\title{
El Sintesista: Contexto, Evolución y Nuevas Perspectivas de Desarrollo
}

Daniel Gómez ${ }^{1}$

David Sánchez ${ }^{2}$

\section{Resumen}

Luego de una revisión histórica de la evolución del sintetizador y del estudio de las aproximaciones cognitivas de la enseñanza musical, se enuncian y definen factores que son importantes tener en cuenta en el diseño y la construcción de nuevos instrumentos e interfaces para la síntesis de audio. Multidimensionalidad, espacios tímbricos, retroalimentación multisensorial y expresividad se muestran como aspectos decisivos en la construcción de sintetizadores, formando el paradigma a seguir por la nueva generación de diseñadores de instrumentos digitales. Su reto principal es desarrollar herramientas cada vez más intuitivas que estimulen y faciliten el proceso de acercamiento del músico a la síntesis de audio.

\section{Palabras clave}

Cognición, informática musical, interfaz, sintesista, síntesis de audio.

1 Facultad de Ingeniería, Universidad ICESI, Cali-Colombia, dgomez@icesi.edu.co

2 Facultad de Artes y Humanidades, INSTITUTO TECNOLÓGICO

METROPOLITANO, Medellín-Colombia, davidsanchez@itm.edu.co

Fecha de recepción: 15 de Marzo de 2010

Fecha de aceptación: 24 de Octubre de 2010

Rev. Tecno Lógicas No. 25, ISSN 0123-7799, Diciembre de 2010, pp. 11-29 


\section{Abstract}

After a historical review of the synthesizer's evolution and the study of cognitive approaches of musical teaching, some factors are stated and defined that are important to consider for the design and construction of new instruments and audio synthesis interfaces. Multidimensionality, timbre spaces, multi sensorial feedback and expressiveness appear as decisive aspects in the construction of synthesizers, shaping the paradigm to be followed by the new generation of digital instrument designers. Their main challenge is to develop more intuitive and stimulating tools that ease the approaching process of musicians to sound synthesis.

\section{Keywords}

Cognition, interface, music informatics, sound synthesis, synthesist. 


\section{CONTEXTO}

El sintetizador es una herramienta diseñada para generar sonidos y controlar detalladamente su timbre, utilizando elementos sencillos interconectados entre sí en esquemas especiales dependiendo del tipo de síntesis (Loy, 2005). Moore define el timbre como "El atributo de la sensación auditiva en términos del cual un escucha puede juzgar que dos sonidos presentados similarmente con la misma amplitud y afinación son diferentes" (Moore, 2003). Con el tiempo los elementos de la síntesis han aumentado en cantidad y han ido refinándose, hasta el punto de tener unos elementos estandarizados como osciladores, osciladores de baja frecuencia (LFO), filtros, envolventes, mezcladores de señal, moduladores de anillo, entre muchos otros.

Un sonido con un timbre específico se logra entonces conectando dichos elementos de entre sí y modificando los valores de sus variables. De esta manera, los diferentes tipos de síntesis son diferentes maneras recursivas y esquemáticas de conectar estos elementos básicos y se diferencian entre sí porque tienen una aproximación distinta a la generación del timbre. Los tipos de síntesis pueden clasificarse en síntesis aditiva, sustractiva, FM, granular, entre otras. (Roads, 1996).

Desde la primera formulación de módulos electrónicos de síntesis en la década de los sesentas (Moog, 1965), los instrumentos que realizaban síntesis aditiva y sustractiva consistían en un gran cajón de madera que tenían instalados dichos módulos. Preparar el sintetizador para generar un sonido consistía en realizar conexiones entre los módulos utilizando cables de audio y en ajustar los potenciómetros de cada uno de éstos. Esta red de conexiones entre módulos de un mismo sintetizador se conoce como patch. Un patch define entonces el flujo de electricidad a través de los módulos, lo que a su vez produce un tipo de sonido en particular; es por eso que en cierta literatura se confunde el uso de "patch" como una red de conexiones y "patch" como pre configuración de un sonido; ambos están correctos (Pinch \& Trocco, 2004).

Este gran cajón con su red de cables y potenciómetros (Chadabe, 2000) evolucionó a una caja de madera más pequeña 
con las conexiones ya escondidas, aun llena de potenciómetros y con un teclado de piano en la parte inferior. Este nuevo modelo es el paradigma de lo que hoy se conoce como sintetizador y el primero de su especie fue el Minimoog. Al ocultar los cables del patch detrás del cajón, el sintetizador deja de parecer un equipo para expertos y empieza a mimetizarse entre la familia de los instrumentos musicales tradicionales. Para la operación del sintetizador, el ocultar los cables del patch supone que todas las conexiones son posibles y son mediadas por nuevos elementos incorporados al cajón: interruptores. Aunque el Minimoog es más simple a los ojos, tiene grandes desventajas con sus predecesores modulares, ya que en realidad no todas las conexiones son posibles (por ejemplo no se puede realizar síntesis FM o modulación de anillo) y las posibles son invisibles a los ojos. Podemos decir que de alguna manera la red del patch hace que se vea mucho más confuso en primera instancia, pero en la práctica, conocer la ruta de la señal a través del equipo, permite un control más intuitivo (Choi et al., 1995).

En la década de los setentas llegan los micro-controladores digitales, y nuevos personajes como Dave Smith y John Chowning se embarcan en el diseño de nuevos sintetizadores y en la creación de nuevos paradigmas en el área de la síntesis de audio. Por su parte, Dave Smith ayuda a solucionar el tema de la creación de patches, a nuevos usuarios de sintetizadores, diseñando módulos de presets para éstos. Chowning descubre y patenta un nuevo tipo de síntesis de audio llamado Síntesis FM (Chowning, 1973). Los presets como bien lo dice Smith son ayudas para sintesistas (término que se usará a lo largo de este texto para referirnos a los músicos que realizan síntesis de audio y tocan sintetizadores); esta ayuda les permite almacenar un patch que han creado tras gran esfuerzo. Un preset es entonces la colección de los valores de cada una de las variables del patch de un sintetizador y preconfigura el tipo de sonido que el sintetizador genera: al pasar entre un preset y otro se está pasando entre un patch y otro o, lo que es lo mismo, entre un sonido y otro, haciendo más sencillo el uso del sintetizador y abriendo la posibilidad de almacenar nuestros propios sonidos. Por el lado de la síntesis FM, Chowning comprobó que aún había terrenos inexplorados en la creación de timbres y 
que podían existir nuevos métodos de síntesis, más económicos, donde se usen menos elementos que en la síntesis aditiva y sustractiva para obtener sonoridades complejas. Yamaha utiliza la tecnología de síntesis FM y lanza el sintetizador digital DX7 y logra que todas las tarjetas de sonido integradas a los computadores generen los sonidos usando síntesis FM (Smith, 2007).

En la década de los ochentas, los controles del sintetizador son minimizados al extremo, por ejemplo el sintetizador DX7 no tiene cables ni potenciómetros en su interfaz física para modificar los patches, sino que su manejo favorece principalmente el uso de presets, a través de unos cuantos botones como herramienta principal para modificar los sonidos. El sintetizador viene con una lista de decenas de sonidos preconfigurados y su modificación es bastante difícil para los intérpretes, es bien conocida la leyenda de que de cada 10 DX7 que llegaban a reparación 9 de ellos no tenían ninguna modificación de los patches originales. Visto de otra manera el 90\% de los usuarios del DX7 estaban usando los mismos sonidos para realizar sus composiciones y producciones musicales.

La llegada de los sintetizadores digitales y los computadores personales supone una segunda revolución para los sintesistas, pues el costo de producción de nuevos sintetizadores y módulos de procesamiento de audio disminuye, favoreciendo esto la masificación de los sintetizadores de todos los tipos. Por otro lado, Dave Smith unificó el protocolo de comunicación, entre sintetizadores y unidades de procesamiento de audio, y de sincronización creando el protocolo Musical Instrument Digital Interface más conocido como MIDI (Smith, 1981). La mayoría de productores de equipos musicales adoptan este protocolo y el MIDI se convierte en el estándar de comunicación entre ellos. La computadora personal empieza a perfilarse como una nueva herramienta poderosa capaz de grabar, almacenar y reproducir MIDI y muestras de audio. Comienza una lenta migración de los estudios de grabación y sus utilidades al computador personal y nace lo que hoy se conoce como estaciones de audio digital o DAW por sus siglas en inglés (Digital Audio Workstations).

La velocidad de los procesadores digitales permite experimentar nuevas técnicas de síntesis que antes requerían una 
gran cantidad de módulos físicos $\mathrm{y}$ ahora solo requieren habilidades de programación. Comienza un proceso de migración de la tecnología análoga a la tecnología digital. Es aquí cuando surgen nuevos métodos de síntesis como la síntesis granular, que combina los elementos básicos de síntesis con procesos de reproducción de pequeñas muestras de audio digital para crear una nueva gama de sonidos. Hay también un aumento en la capacidad de procesamiento de los computadores y aparece una cuarta familia de síntesis que utiliza el modelado acústico de cuerdas en vibración y tubos resonantes para recrear artificialmente su fenómeno físico y así generar sonidos no por imitación (como en la síntesis aditiva y la FM) sino por modelación.

En la década de los noventas la capacidad de los computadores llega casi a cumplir la promesa de la generación y procesamiento de audio en "tiempo real" y viene acompañada de software que revoluciona el ejercicio de la música por computador y crea un nuevo paradigma. Es la familia de software MSP que retoma la idea de módulos interconectados formando patches, pero los módulos son representados virtualmente en la pantalla del computador. Se habla del Max/MSP, el Reaktor y el PureData entre otros. En este momento se empiezan a borrar los límites para la construcción de sintetizadores virtuales utilizables en tiempo real. La atención se vuelca sobre el diseño de herramientas que aumentan las posibilidades de los instrumentos existentes o que por medio de sensores exploran el control de la síntesis de audio a través de diversos gestos motrices.

Durante todo el proceso de evolución de los sintetizadores, la síntesis ha sido un proceso complejo y distante a músicos e intérpretes. A pesar de estar en esta época de expansión de las posibilidades de construcción de sintetizadores e interfaces, el aprendizaje de la síntesis de audio y la interacción con el timbre siguen siendo actividades de un orden superior al simple acto de hacer sonar un instrumento tradicional. El problema es que dicha expansión se ha centrado principalmente en el desarrollo tecnológico: por un lado está el aumento de las posibilidades algorítmicas para la generación del sonido, por otro lado se encuentra el desarrollo de interfaces centradas en la 
experimentación con sensores y motricidad, y finalmente el enlace entre algoritmos y sensores (mapping). Este enfoque en el aspecto tecnológico deja a un lado la reflexión sobre cómo se da la aproximación de un músico a la síntesis, es por esto que muchos de los instrumentos que se diseñan no trascienden porque no tienen en cuenta factores como el aprendizaje y el desarrollo de un control intuitivo. Pareciera como si a pesar de haber evolucionado tanto la tecnología tuviéramos los mismos problemas que encontraba un sintesista al enfrentarse a los primeros y voluminosos sintetizadores análogos de los años sesenta.

Esta problemática lleva a hacer una revisión de diversas aproximaciones pedagógicas de la música y el desarrollo instrumental que pueda dar luces para el planteamiento de métodos eficaces para el diseño y la construcción de nuevos instrumentos musicales.

\section{REVISIÓN DE LAS METODOLOGÍAS PEDAGÓGICAS TRADICIONALES DE LA MÚSICA}

Como lo ilustra David Hargreaves, los estudios acerca de la enseñanza musical en el siglo $\mathrm{XX}$ han arrojado más aproximaciones pedagógicas que teorías como tales, ya que se han desarrollado más desde la práctica que desde la teoría psicológica y sociológica (Hargreaves, 1998).

El área privilegiada de reflexión por parte de los educadores ha sido la iniciación musical; el primer acercamiento a este universo sonoro es, sin duda, la preocupación principal de muchos pedagogos. Los métodos desarrollados a partir de esta reflexión se pueden dividir en dos categorías: activos e instrumentales. Los métodos activos, como Dalcroze y Willems, fueron predominantes en las décadas de los cuarenta y los cincuenta y daban prioridad al movimiento corporal, a la entonación de sonidos y a la danza. Los métodos instrumentales, como Orff y Kodály, han sido los más estudiados y aplicados desde mitad de siglo y utilizan como recurso principal la ejecución rítmica y vocal en ensamble, y el desarrollo del oído interior mediante repertorio vocal tradicional, respectivamente. 
$\mathrm{Si}$ bien estos métodos son usados en la mayoría de instituciones actuales, hay una falta de articulación y comunicación entre educadores, los cuales tienen iniciativas y estrategias pedagógicas efectivas e innovadoras, pero que son difíciles de sistematizar.

Hasta la primera mitad del siglo XX predominaban las formas tradicionales de enseñanza musical. Estas formas estaban influenciadas por las técnicas pedagógicas conductistas, en donde a partir de la trasmisión de leyes generales de la teoría musical se comenzaba a construir luego el conocimiento más fino, esto es, mediante procedimiento deductivo; también se caracterizaban por el ordenamiento riguroso de los niveles de aprendizaje y de los esquemas evaluativos.

A partir del año 1960 se han ido incorporando a la mezcla de enseñanza formas progresistas. Inspiradas en el legado de Rousseau y Dalcroze, que daba un lugar especial a la experiencia musical intuitiva, el aprender mediante la exploración era ya más importante que simplemente recibir órdenes, lo que llevó a flexibilizar niveles y parámetros de evaluación y enseñanza.

La mezcla pedagógica que utilizan los maestros actualmente utiliza en mayor o menor medida formas tradicionales y formas progresistas. No se podría emitir un juicio acerca de cuáles son mejores, pero si se podría decir cuáles son más acertadas según los objetivos.

Las formas tradicionales de enseñanza son efectivas para el desarrollo de destrezas de ejecución y conceptos musicales básicos como notación, ritmo, entonación y principios de construcción armónica y contrapuntística. Las competencias de un nivel más avanzado, como la composición e improvisación, escapan al método deductivo de enseñanza, siendo más pertinentes a las formas progresistas.

Es por ello que no se puede desarrollar la misma mezcla pedagógica en todos los tipos de música o etapas del aprendizaje musical, hay que proceder según el tipo de competencias y necesidades particulares de cada género o expresión, y ser pertinente al momento o etapa de dicho aprendizaje.

Tanto la iniciación musical como el aprendizaje instrumental han sufrido a lo largo de las últimas décadas transformaciones de 
los paradigmas que giran en torno a las reflexiones pedagógicas. En 1986 David Hargreaves concluía en su libro "Música y desarrollo psicológico" que a pesar de grandes avances en materia, como por ejemplo la teoría cognitivo-evolutiva de Piaget, todavía no existía registro de educadores musicales que se hubiesen atrevido a articular aproximaciones psicológicas o teorías de procesos cognitivos de aprendizaje a su que hacer (Hargreaves, 1998). Ahora, casi 25 años más tarde, hay algunas instituciones como la ESCOM (European Society for the Cognitive Sciences of Music) y la ICMPC (International Conference of Music Perception and Cognition) enfocadas en dicha temática, pero el interés general en el medio es insuficiente.

\section{COMPARACIÓN DE LOS PROCESOS DE APRENDIZAJE DEL SINTETIZADOR CON LOS PROCESOS DE APRENDIZAJE DE INSTRUMENTOS MUSICALES TRADICIONALES}

Cuando se aborda el tema de la educación en síntesis de audio, se enfrenta a varias problemáticas ajenas al de la educación de instrumentos musicales tradicionales. La diferencia se origina a partir de la naturaleza particular de estos instrumentos como tales, y ésta modifica necesariamente la actitud que alumnos y pedagogos deben tener frente al proceso de enseñanza y aprendizaje.

El docente de instrumentos tradicionales busca que el músico desarrolle capacidades motrices que le permitan hacer "interpretaciones" acertadas de lo que el compositor plasmó en la partitura. Estas partituras son como el "manual a seguir por el intérprete" para la recreación de la obra, y traen "instrucciones" que van desde las más objetivas, como altura y ritmo, pasando por unas menos objetivas, como tempo, articulación y dinámica, hasta las más subjetivas, como fraseo, color y carácter. Es por esto que se forma al alumno físicamente para que pueda reproducir con exactitud las más objetivas, e intelectualmente para que pueda decidir con buen gusto y fundamento estilístico las más subjetivas (Ferguson, 1963). 
La música escrita para sintetizadores ha tenido dificultades de notación unificada que pueda servir para la generación de partituras. Esta problemática fue descrita por Thom Holmes en relación a la música electrónica: "Aún las obras que son trascripciones de composiciones cromáticas convencionales no pueden estar plasmadas enteramente en el papel, ya que los elementos de la instrumentación electrónica, procesamiento de audio e interpretación, desafían la estandarización" (Holmes, 2002).

Es paradójico ver como los sintetizadores, en cuanto máquinas tecnológicas, pueden agregar gran profundidad y expresividad a los elementos subjetivos de la música: paisajes sonoros nunca antes imaginados, mutaciones tímbricas que envidiaría cualquier orquesta, planos dinámicos finos y extremos, etc.; son un recurso tal que incluso a los elementos más objetivos de la música, como ritmo y afinación, les puede imprimir también riqueza y profundidad: afinación intratonal, microritmos, etc. La técnica motriz no impone ya sus límites, éstos están justo donde terminan la imaginación y la destreza intelectual (Winkler, 1999).

Las infinitas y exquisitas posibilidades sonoras, hacen del sintetizador un elemento más apto para la exploración que para la reproducción (Holmes, 2002). Es por eso que es más pertinente a los métodos progresistas de enseñanza, que estimulan el sentimiento musical y la creación, que a los tradicionales.

La enseñanza de la síntesis de audio tendría como fin entonces dar herramientas al intérprete para poder llevar a cabo dicha exploración. La primera de ellas sería conocer y apreciar el sonido y sus características. Siendo la mutación tímbrica el mayor potencial de todo sintetizador, el conocimiento físico del sonido $\mathrm{y}$ sus propiedades es el punto de partida de todo desarrollo posterior. La segunda herramienta sería conocer la forma en que el sintetizador genera y modifica el sonido -como opera internamente- y la manera de usar controles externos para manipular dichas operaciones. Luego de comprender la naturaleza del sonido, y de entender el modus operandi, vendría un momento de vivenciar el sintetizador en todas sus dimensiones; siendo los procesos de síntesis únicos a su especie y diferentes a todo proceso tímbrico acostumbrado en la orquestación tradicional, la única 
forma de entenderlos a pleno es experimentándolos, puesto que una descripción técnica y abstracta (punto inicial de las técnicas deductivas de aprendizaje) no nos diría nada de cómo percibimos realmente el timbre y sus mutaciones.

En los instrumentos tradicionales también hay un proceso similar de síntesis, en este caso acústica, la diferencia está en que es un proceso visible, donde hay una correspondencia directa entre el gesto físico y el sonido resultante (Garnett \& Goudeseune, 1999) (la vibración de una cuerda, el golpe de un redoblante, etc.). Del mismo modo, cambios sutiles en la manera de tocar generan diferencias sutiles en el sonido, (el ángulo del dedo en una cuerda de guitarra, la mayor o menor presión del arco en una cuerda de violín, etc.). Los procesos cognitivos comienzan justo cuando hay una conciencia de la relación gesto-resultado, es aquí cuando se amplían las posibilidades de control e interpretación.

La arquitectura de los sintetizadores contrasta entonces con la

de los instrumentos tradicionales generando problemáticas técnicas, musicales y cognitivas que es necesario tomar en cuenta para hacer revisión del modo a proceder en el diseño de nuevos instrumentos.

Hay una predisposición evidente del músico de conservatorio al uso de sintetizadores, esto se da en parte por el tradicionalismo que se maneja en las instituciones y por el poco espíritu creativo y experimental que tienen los intérpretes hoy día; pero la responsabilidad de esta distancia no reside del todo en músicos e instituciones (Russ, 2004).

\section{FACTORES IMPORTANTES EN EL DISEÑO DE NUEVOS INSTRUMENTOS DIGITALES}

El desarrollo de sintetizadores comerciales ha estado más en manos de técnicos e ingenieros que de músicos y artistas, esto ha llevado a que haya habido más preocupación por el desarrollo de algoritmos y optimización de la estructura operativa interna, que por resolver la pregunta de cómo el usuario va a utilizar estos para comunicar sentimientos, sensaciones y programas de igual forma que lograr expresividad musical (Seago, 2004). Sin embargo, hay 
una nueva generación de diseñadores de instrumentos de síntesis de audio que ha buscado solucionar los problemas cognitivos anteriormente descritos. Esta generación ha fundamentado su labor en temáticas específicas que conforman el paradigma del diseño y construcción de nuevos instrumentos.

\subsection{Multidimensionalidad}

Muchos autores han descrito las limitaciones que tienen los sintetizadores comerciales debido a la interacción poco intuitiva que plantea el diseño de su interfaz (Seago, 2004; Miranda, 2002; Hazel, 1992) y a la complejidad dimensional que supone el acercamiento al control del timbre (Choi et al., 1995) para la mayoría de los métodos de síntesis potentes. Para algunos, las limitaciones dependen del lenguaje, pues el acercamiento de un sintesista al aparato supone una conexión entre las dimensiones perceptivas de cada sintesista (como brillo, densidad, grosor, color del sonido, etc.) con unas dimensiones técnicas en las cuales se escriben los métodos de síntesis (frecuencia, amplitud de la modulación, resonancia, etc.). Como se ha visto antes, esta brecha semántica se acorta por diversos procesos cognitivos principalmente de experimentación, ensayo y error. Para otros autores, la retroalimentación del acto tímbrico (escucha / análisis / manipulación) genera una sobrecarga cognitiva (Garnett \& Goudeseune, 1999) responsable de saturar todo el ancho de banda cognitivo del sintesista y cortocircuitar las posibilidades de generar un conocimiento por ensayo y error debido a la proliferación de variables disponibles incomprendidas.

\subsection{Espacios Tímbricos}

La complejidad multidimensional del timbre es entonces una piedra angular en el diseño de nuevos sintetizadores que acerquen más al usuario a los métodos de síntesis. Las miradas que por más de 40 años se han dado al tema de la complejidad del timbre (Grey, 1975; Wessel, 1979; Hourdin, 1997; Momeni \& Wessel, 2003; Seago et al., 2005) han creado y desarrollado el concepto de 
Espacio Tímbrico como espacios multidimensionales cuyos ejes representan las dimensiones perceptivas del timbre.

Con el tiempo han surgido diversas estrategias que se basan en el concepto de Espacio Tímbrico para manipularlo y a través de él poder disminuir la complejidad percibida por un sintesista. La mayoría de ellas se han materializado en instrumentos para el control de la síntesis de audio. Entre estas estrategias están el uso de algoritmos genéticos para realizar conexiones entre parámetros deducidas por redes neuronales (Horner et al., 1993; Johnson, 1999; Miranda, 2002), el uso de métodos estadísticos para la predicción de la asignación de valores a un sintetizador (Loviscach 2008), la representación geométrica inteligente del espacio tímbrico para realizar interacciones efectivas sobre el mismo usando tabletas digitales (Momeni et al., 2003), el uso de conexiones entre un espacio tímbrico con variables perceptuales y un espacio tímbrico con variables técnicas.

Esta construcción geométrica de la complejidad multidimensional del timbre es entonces el espacio que debe ser estudiado y optimizado por un diseñador de interfaces para síntesis de audio, donde por diversas técnicas se busca acercar el usuario al control del sonido usando diversos tipos de estrategias, dependiendo del contexto, pero siempre favoreciendo la cognición de los instrumentos.

\subsection{Retroalimentación Multisensorial}

El estudio de la cognición de los instrumentos musicales recientemente ha evidenciado la importancia de la retroalimentación multidimensional de los instrumentos musicales hacia los músicos en dimensiones adicionales al sonido. Estímulos multisensoriales entre un instrumento (digital o acústico) y un músico han probado ser factores decisivos en el acercamiento y la adaptabilidad a un instrumento en particular por un intérprete (Jordá, 2005; Reyes, 2004). La háptica es una rama de la Interacción Humano-Computador (HCI por sus siglas en inglés) que estudia el uso y aprovechamiento del sentido del tacto para apoyar la cognición y el control de objetos virtuales; ésta desarrolla estímulos de fuerza, vibración y movimiento desde 
una interfaz hacia un usuario para satisfacer la creación de una imagen cerebral de un objeto virtual (Robles-de-la-torre, 2008). Junto con la retroalimentación háptica existen otras relacionadas con los demás sentidos: visual, cinética y demás dispuestas a apoyar un gesto sonoro. La retroalimentación audio visual ha sido objeto de múltiples y diversos estudios como las teorías de la Audiovisión (Chion, 1990), aplicaciones donde se encuentra toda la familia de instrumentos virtuales de interfaz gráfica y los plugins para el computador (Steinberg 1996; Furse, 2005), donde también encajarían las distorsiones perceptivas de la sinestesia.

\subsection{Expresividad}

Con la tecnología disponible de microprocesadores programables para procesar señales digitales y una amplia gama de sensores, un numeroso grupo de músicos, limitados por las herramientas de síntesis y procesamiento disponibles comercialmente, junto con investigadores de tecnologías de procesamiento de señal, programadores de algoritmos de síntesis y procesamiento y entusiastas experimentadores del sonido, se volcaron a la creación de interfaces sonoras con aplicaciones musicales. Los principios y aplicaciones de esta generación de instrumentos digitales fueron condensados en una conferencia que se inaugura en el 2001 como NIME (New Interfaces for Musical Expression). Con el NIME y sus instrumentos se busca enfatizar en la Expresividad como principal finalidad de un instrumento musical, superando la aplicación tecnológica y la experimentación en el diseño de interfaces. Es interesante resaltar que la proliferación de nuevos instrumentos y nuevas interfaces está más cercana al impulso del hacer que de la reflexión, hecho resaltado por Perry Cook en su presentación principal "Principios para controlar diseñadores de música por computador" en el NIME 2007 (paráfrasis de su artículo "principios para diseñar controladores de música por computador") (Cook, 2001). Este grupo de investigadores y músicos que desarrollan nuevas Interfaces para la expresión musical, ofrecen permanentemente una extensa gama de instrumentos, interfaces, controladores y novedosos acercamientos al desarrollo de la motricidad y de la 
interacción con el sonido (Reyes \& Lawson, 1997; Arcos et al., 1997; Fiebrink et al., 2007).

\section{CONCLUSIONES}

La noción de timbre es compleja, debido a esto, los dispositivos desarrollados para su control heredan esa misma naturaleza de complejidad. Sin embargo, el reto que tienen los diseñadores de nuevos instrumentos es la creación de estrategias contundentes que acerquen los usuarios a los detalles más profundos del timbre, sin sumirlos en niveles de sobrecarga cognitiva.

El diseño de herramientas de síntesis de audio no debe ser trabajado desde un solo punto de vista ya sea tecnológico, ergonómico, pedagógico, entre otros, sino que debe ser un trabajo multidisciplinario que involucre las áreas mencionadas, junto con psicología, interpretación musical, procesamiento de señales, antropología y todas las ramas del conocimiento que puedan aportar ideas para el desarrollo de nuevas herramientas.

Los procesos cognitivos de aprendizaje de los instrumentos musicales tradicionales y la manera como el músico interactúa con ellos, son aspectos fundamentales y determinantes en el diseño y construcción de nuevos instrumentos, ya que algunos aspectos de estos procesos son comunes a los que se llevan a cabo en la interacción con los sintetizadores. Se pueden mencionar las relaciones gesto-resultado tímbrico, que cuando el músico toma consciencia de ellas se amplía la gama de posibilidades y profundidades en la interpretación.

Los factores encontrados por investigadores musicales alrededor del mundo en diversos períodos, como la multidimensionalidad tímbrica, la háptica, la expresividad y la retroalimentación multisensorial, son temas que un grupo de trabajo debe tener siempre en cuenta cuando planeta un proyecto de desarrollo de nuevos instrumentos.

La literatura científica revisada para este artículo describe la evolución de diversos temas que con el paso del tiempo han ido apareciendo en la investigación del diseño de nuevos instrumentos musicales. Sin embargo, algunos de los investigadores autores de 
esta literatura, complementan sus estudios con "decálogos" y "listas de deseos" como guías para futuros diseños. Estas listas no son conclusiones del trabajo en laboratorio, ni afirmaciones fundamentadas en datos científicos, sino reflexiones personales que provienen de la experiencia de los autores en trabajos realizados durante años. Al no existir aún formulaciones científicas que comprendan toda la complejidad de tópicos necesarios para el diseño y construcción de nuevos instrumentos, estas reflexiones son herramientas que pueden usarse para llenar los espacios que aún no son cubiertos por la ciencia.

\section{REFERENCIAS}

Arcos J.L., de Mantaras R.L., and Serra, X. (1997); SaxEx: a Case-Based Reasoning System for Generating Expressive Musical Performances, in Proceedings of 1997 International Computer Music Conference, 329-336, Thessaloniki, Grecia.

Chadabe, J., (2000); The Electronic Century Part III: Computers and Analog Synthesizers. Electronic Musician: Tutorials, 66-70.

Chion, Michel, (1990); La audiovisión, Paidós Comunicación, Barcelona.

Choi, I., Bargar, R., Goudeseune, C., (1995); A Manifold Interface for a High Dimensional Control Space, Actas de la Conferencia Internacional de Música por Computador ICMC, 1-2, Banff, Canada.

Chowning, J., (1973); The Synthesis of Complex Audio Spectra by Means of Frequency Modulation, Journal of the Audio Engineering Society 21:526-534.

Cook, P., (2001); Principles for designing computer music controllers, NIME workshop.

Ferguson, H. (1963); Style and Interpretation, 2a Edición, Volumenes 1-6, Oxford University Press, London, England.

Fiebrink, R., Wang, G., Cook, P., (2007); Don't forget the Laptop: Using Native Input Capabilities for Expressive Musical Control, Proceedings NIME 2007, 164-167.

Furse, R. (2005); LADSPA, Available: http://www.ladpsa.org, last time checked: 2005 July 7 th. 
Garnett G., Goudeseune C., (1999); Performance Factors in Control of High-Dimensional Spaces, International Computer Music Conference, Beijing, China.

Grey, J.M., (1977); Multidimensional perceptual scaling of musical timbres, Journal Acoustical Society of America, 1270-1277.

Grey, J. M., (1975); An Exploration of Musical Timbre, Ph.D. Thesis, Dept. of Psychology, Stanford University.

Hargreaves, D.J., (1998); Música y desarrollo psicológico, $1^{\text {a }}$ Edición, 241, 248, Graó, Barcelona, España.

Hazel, P., (1992); Synthesizers: Interface, Design and Development, M.Sc dissertation.

Holmes, T., (2002); Electronic and Experimental Music, 2a Edición, Routledge, New York, United States of America.

Horner, A., Beauchamp, J. and Haken, L., (1993); Machine tongues XVI: genetic algorithms and their application to FM matching synthesis, Computer music Journal, 17:4, 17-29.

Hourdin, C., Charbonneau, G. Moussa, T., (1997); A multidimensional scaling analysis of musical instruments' time varying spectra, Computer music journal, 21:2, 40-55.

Jordà, S. (2005); Digital Luthiere, Tesis Doctoral, Departament de Tecnologia, Universitat Pompeu Fabra, Barcelona, España, 19-27.

Johnson, C.G., (1999); Exploring the sound-space of synthesis algorithms using interactive genetic algorithms, AISB'99 Symposium on Musical Creativity, Edinburgh.

Loviscach, J., (2008); Programming a Music Synthesizer through Data Mining, Proceedings of NIME 2008, 221-224.

Loy, G., (2005); Sound Synthesis, In: Musimathics Vol II by MIT Press, Cambridge, MA, USA.

Miranda, E., (2002); Computer Sound Design: Synthesis Techniques and Programming, Publisher: Focal Press, Second edition.

Momeni, A., Wessel, D., (2003); Characterizing and controlling musical material intuitively with geometric models, Proceedings Conference on New Interfaces for Musical Expression NIME, Montreal, Quebec, Canada. 
Moog, R.A., (1965); Voltage-Controlled Electronic Music Modules, AES Convention, October, 346.

Moore, B., (2003); An introduction to the psychology of hearing, Academic Press. Boston, Estados Unidos.

Pinch, T., Trocco, F., (2004); Analog Days: The Invention and Impact of the Moog Synthesizer, 53-69, Harvard University Press, Cambridge, MA, USA.

Puckette, M.S., (1996); Pure Data, in International Computer Music Conference ICMA, Hong Kong, 269-272.

Reyes, J., (2004); Elements and Introduction to Physical Interaction I Introducción y Elementos para Interacción Física: Tutorías para Artistas y Músicos (mostly in spanish) disponible en línea: http://www.maginvent.org/articles/pidht/pidtoot/pidtoot.html.

Reyes J., Lawson C., (1997); Another Approach to Expression with Algorithms, Bogotá, Colombia, MOX - Computación Avanzada en Ingeniería, Universidad de Los Andes.

Roads, C., (1996); The Computer Music Tutorial, 85-114. MIT press, Cambridge, MA, USA.

Robles-De-La-Torre G., (2008); Principles of Haptic Perception in Virtual Environments. In Grunwald M (Ed.), Human Haptic Perception, Birkhäuser Verlag.

Russ, M., (2004); Sound Synthesis and Sampling, 2a Edición, 3, Focal Press, Burlington, United States of America.

Seago, A., Holland, S., Mulholland, P., (2005); Towards a Mapping of Timbral Space, Conference on Interdisciplinary Musicology (CIM05), Montreal, Canada.

Seago, A. (2004); Electronic Sound Synthesizer Interface Design.

Seago, A., Holland, S., Mulholland, P., (2004); A Critical Analysis of Synthesizer User Interfaces for Timbre. HCI 2004: Design for Life, Leeds, British HCI Group.

Smith, D., (2007); An afternoon with Dave Smith, Episode One, New Island Media. Disponible en línea:

http://video.google.com/videoplay?docid=-250309729795719271\#

Smith, D., (1981); MIDI: A Network Protocol for Musical Instrument Control. Actas de la Sociedad de Ingeniería de Audio, New York, USA. 
Steinberg A.G., (1996); Virtual Studio Technology, Disponible en línea: http://ygrabit.steinberg.de, last time checked: 2005 July 7 th.

Wessel, D.L., (1979); Timbre space as a musical control structure, Computer music Journal, 3(2), 45-52

Winkler, T., (1999); Composing Interactive Music, $2^{\text {a }}$ Edición, 34, MIT Press, Cambridge, London, England. 
